\title{
Prophylactic use of exogenous melatonin and melatonin receptor agonists to improve sleep and delirium in the intensive care units: a systematic review and meta-analysis of randomized controlled trials
}

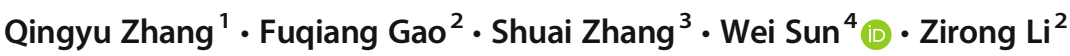

Received: 18 October 2018 / Revised: 21 February 2019 / Accepted: 21 March 2019 / Published online: 22 May 2019

(C) Springer Nature Switzerland AG 2019

\begin{abstract}
To investigate the efficacy of exogenous administration of melatonin and melatonin receptor agonists for the improvement of delirium, sleep, and other clinical outcomes of subjects in the intensive care unit (ICU). We carefully searched three electronic databases, i.e., Pubmed/Medline, Embase, and Cochrane library, to retrieve randomized controlled trials (RCTs) administrating melatonin or melatonin receptor agonists to adult subjects admitted to the ICU. Useful data such as the prevalence of delirium, duration of sleep, number of awakenings per night, duration of mechanical ventilation, and ICU stay as well as in-ICU mortality were extracted and pooled by using a random effect model. Eight RCTs were included in the qualitative analysis. Administration of exogenous melatonin and melatonin receptor agonists was associated with a trend towards elongated duration of sleep (pooled weighted mean difference/ $\mathrm{WMD}=0.43 ; 95 \%$ confidence intervals $/ \mathrm{CIs},-0.02 \sim 0.88, p=0.063$ ) and could decrease the number of awakenings per night (pooled WMD $=-2.03 ; 95 \% \mathrm{CIs},-3.83 \sim-0.22, p=0.028$ ). Meanwhile, participants in the treatment group showed a significantly reduced prevalence of delirium (pooled risk ratio/ $\mathrm{RR}=0.49 ; 95 \% \mathrm{CIs}, 0.28 \sim 0.88, p=0.017$ ) and duration of ICU stay (pooled WMD $=-0.32 ; 95 \% \mathrm{CI},-0.56 \sim 0.07, p=0.002$ ) in comparison with those in the control group. Exogenous administration of melatonin and melatonin receptor agonists could improve the sleep of subjects in the intensive care units, which may play an important role in decreasing the prevalence of delirium and shortening duration of ICU stay.
\end{abstract}

Keywords Intensive care unit $\cdot$ Delirium $\cdot$ Melatonin $\cdot$ Circadian rhythms $\cdot$ Sleep deprivation $\cdot$ Meta-analysis

Qingyu Zhang and Fuqiang Gao are joint first authors.

Electronic supplementary material The online version of this article (https://doi.org/10.1007/s11325-019-01831-5) contains supplementary material, which is available to authorized users.

Wei Sun

sunwei20180101@163.com

1 Department of Orthopedics, Shandong Provincial Hospital affiliated to Shandong University, No.324, Road Jing Wu Wei Qi, Jinan 250021, Shandong, China

2 Beijing Key Laboratory of Immune Inflammatory Disease, China-Japan Friendship Hospital, 2 Yinghuadong Road, Chaoyang District, Beijing 100029, China

3 Department of Neurology, Affiliated Hospital of Yangzhou University, Yangzhou University, 368 Hanjiang Road, Yangzhou 225100, China

4 Beijing Key Laboratory of Immune Inflammatory Disease, China-Japan Friendship Hospital, Peking Union Medical College, 2 Yinghuadong Road, Chaoyang District, Beijing 100029, China

\section{Introduction}

Melatonin is the major natural neurohormone produced by the pineal gland and known to manifest diverse physiologic actions including anti-inflammatory, antitoxic, hypnotic, anticonvulsive, sedative, and analgesic properties as well as an effect on the regulation of circadian rhythm [1]. Secretion rhythm of melatonin in patients admitted to the intensive care unit (ICU) has been demonstrated to be severely suppressed due to notorious environmental factors such as noise, 24-h artificial light and limited natural light exposure, greater severity of illness compared with the general wards, and universal application of sedative and narcotic drugs, which may further contribute to increased awakenings per night as well as shortened duration and compromised quality of sleep [2,3]. Meanwhile, sleep deprivation, affected sleep-wake cycle, and abnormal melatonin excretion are associated with the occurrence of delirium, a frequently encountered organ dysfunction 
in critically ill patients with an incidence ranging from 45 to $87 \%[4,5]$. Delirium is characterized by consciousness disorder with cognitive change (hyperactive, hypoactive, or mixed form) and is a well-known risk factor for prolonged duration of mechanical ventilation and ICU stay, higher mortality, greater risk of cognitive sequelae, and more hospital costs [5-7]. It has been demonstrated that sleep deprivation and delirium affect the same region (mainly the prefrontal and parietal cortexes) of the central nervous system with similar neurohormonal changes (decreased acetylcholine or increased dopamine levels) [2].

In comparison with melatonin, melatonin receptor agonists (e.g., ramelteon and tasimelteon) have a longer half-life and higher absorptivity [8]. Clinical trials have suggested that exogenous melatonin and melatonin receptor agonists can be used to treat insomnia and anxiety without inducing any adverse effect on psychomotor and cognitive performance [8-10]. Moreover, unlike conventional sedatives, melatonin does not worsen hypoxemia or respiratory responses at night $[10,11]$. Considering the potential role of melatonin in the development of sleep disorder and delirium in the ICU, the prophylactic use of melatoninergic agents is emerging as an attractive therapeutic supplementary option. However, studies exclusively assessing the impact of exogenous administration of melatonin or melatonin receptor agonists on patients in ICU are few and small scale; meanwhile, study designs and applied evaluation indicators of these investigations are heterogenous, which restrict the generalizability of their conclusion $[12,13]$.

A former systematic review [12] attempting to address the impact of melatonin for the improvement of sleep in adults admitted to ICU was published in 2018. However, we noticed that authors only retrieved four studies including one lowquality randomized controlled trial/RCT [14] and did not conduct quantitative analysis. On the other hand, multiple eligible randomized controlled trials and some important outcomes such as delirium occurrence and sleep duration were neglected by this article. Therefore, we performed this updated systematic exploration to further examine whether the routine administration of melatonin and melatonin receptor agonists was effective for improving sleep, delirium, and prognosis of subjects in ICU.

\section{Methods}

The checklist of the Preferred Reporting Items for Systematic Reviews and Meta-Analyses (PRISMA) statement was followed in the conduction of this investigation [15]. Processes including electronic searches, reference lists screening, study selection, data extraction, assessment of risk of bias, and summary estimate pooling were performed by two investigators (QY Zhang and FQ Gao) independently. Any disagreement was resolved by discussion and consensus. Due to the fact that all required data were retrieved from published articles, informed consent or ethics approval was not required.

\section{Literature search}

We searched three electronic databases, i.e., Pubmed/Medline, Embase, and Cochrane library (from inception to August 30, 2018), by using queries combined by the following terms: (melatonin OR ramelteon OR tasimelteon OR agomelatine OR TIK 301 OR melatonin receptor agonist) AND (delirium OR sleep) AND (intensive care unit OR ICU). Reference lists of classic and related literatures (especially reviews and metaanalyses) about the use of melatonin and melatonin receptor agonists in ICU patients were also carefully screened to retrieve additional eligible articles not identified by electronic database searching. There were no language or data restrictions.

\section{Inclusion and exclusion criteria}

Studies were included in the qualitative analysis if they confirmed the criteria listed as below: (1) randomized controlled clinical trials; (2) subjects were those admitted to intensive care unit with any diagnosis; (3) melatonin or melatonin receptor agonists were prophylactically used after admission to ICU; and quantitative analysis if (4) sleep, delirium, and other clinical variables were provided as endpoints. Exclusion criteria of this investigation were as follows: (1) patients who had already received melatonin or melatonin receptor agonists prior to the admission to ICU; (2) study protocols, letters, correspondences, and conference addressing; and (3) animal studies. We first removed redundant and unrelated records by reading the titles and abstracts. Then, the full texts of the remainders were downloaded to confirm their eligibility based on the above criteria.

\section{Data collection}

The following information were collected from the included articles: first author's family name, year of publication, study design, inclusion criteria of subjects, type and dosage of used agent, the number of participants in the treatment group and the control group, demographic and clinical characteristics of enrolled subjects (e.g., age, sex, admission diagnosis, and application of mechanical ventilation), endpoint parameters of interest (e.g., delirium incidence and duration, duration of mechanical ventilation and ICU stay, mortality, and related adverse events), and adopted evaluation methods. Continuous variables were presented as means with standard deviations (SDs) and, where not available, we approximated them using sample sizes, medians, ranges, and interquartile ranges according to Wan and Luo's methods $[16,17]$. Meanwhile, 
binary variables were expressed through the number of events and frequency in percentage.

\section{Risk of bias assessment}

A tool [18] provided by the Cochrane handbook was used to appraise the risk of bias of included studies, which consisted of seven domains, i.e., random sequence generation, allocation concealment, blinding of participants and personnel, blinding of outcome assessment, reporting of incomplete outcome data, selective reporting, and other biases. Each item was judged as low, unclear, and high risk according to the information provided by original articles. Results of the methodological quality assessment were summarized in a standardized figure.

\section{Statistical analysis}

Stata for Windows (12.0 Version, StataCorp, College Station, TX, USA) was implemented to conduct the quantitative analysis and produce forest plots. Evaluation parameters of interest were combined by using a random effect model. For binary data, pooled risk ratio (RR), as well as related $95 \%$ confidence intervals (CIs), was adopted, and a pooled $95 \%$ CI not covering 1 indicated a significant difference between two groups; meanwhile, pooled weighted mean difference (WMD) or standardized mean difference (SMD), as well as related 95\% CIs, was used to evaluate continuous data, and a 95\% CI not covering 0 revealed a statistical difference. Heterogeneity degree across included studies was appraised using $I^{2}$ statistic, with an $I^{2}$ of $>50 \%$, demonstrating a statistically significant heterogeneity. Then, we conducted sensitivity analyses by omitting studies one by one to assess the stability of results and performed subgroup analyses to address the source for heterogeneity. In the event that data aggregation was infeasible, we performed qualitative analysis.

\section{Results}

\section{Description of included studies}

A total of 158 records were screened in the electronic database searching process and 26 additional ones were retrieved by screening reference lists of related articles. Eventually, eight studies were considered to be qualified for the quantitative analysis [4, 19-25]. One RCT [14] that had been incorporated into the previous systematic review [12] was not included in this study for the following reasons: (1) this study did not exclusively apply melatonin to subjects in the treatment group; (2) there were patients who had melatonin administration stopped before the end of the study; and (3) only one subject was available for the analysis of sleep time in each group. The inclusion processes and reasons for exclusion were depicted in Fig. 1.

All eight studies were blinded randomized controlled trials published in English and among them, one [19] was tripleblinded, six [4, 20-22, 24, 25] were double-blinded, and one [23] claimed to be rater-blinded. Sample sizes of included studies ranged from 20 to 88 with a total of 409 participants. Of this total, 201 subjects were assigned to the intervention group and 208 to the control group. Six [4, 20-22, 24, 25] studies used melatonin (ranges from 1 to $30 \mathrm{mg}$ every day) in the intervention groups and two $[19,23]$ investigated the effect of ramelteon ( $8 \mathrm{mg}$ every day). Four [20, 21, 24, 25] studies only included mechanically ventilated patients, two $[4,19]$ studies enrolled a mixture of both ventilated and nonventilated subjects, and one [23] study did not specify whether recruited patients received mechanical ventilation or not. Patients' admission diagnoses were reported in seven [4, 19-24] studies. Seven [4, 19, 20, 22-25] studies administrated medications with a single dose in the evening and one [21] study was given interventions twice a day at $8 \mathrm{pm}$ and midnight, respectively. The main characteristics of selected studies were listed in Table 1.

As for the risk of bias of the included articles, two $[4,19]$ high-quality double-blinded or triple-blinded studies were rated as low risks in all seven items. The study conducted by Hatta et al. [23] was judged with high risk in binding of participants and personnel because nurses who managed the study medication were unblinded to the grouping, and the appearance of placebo in the control group did not match the appearance of used active agent. One study [25] was judged as unclear risk of bias in incomplete outcome data because lost to follow-up was not clearly reported. No clinical trial registration number or document was identified in two studies [22, 25]; therefore, they were considered with an unclear risk of selective reporting bias. Another bias was unable to be clearly assessed in Bourne et al.'s and Mistraletti et al.'s studies because baseline characteristics were not comparable (e.g., male and age) [21, 24]. Dianakthah et al. [20] provided no details about the allocation concealment and blinding of outcome assessment. Results of the risk of bias assessment were summarized in Fig. 2.

\section{Quantitative analysis}

\section{Prevalence of delirium}

Three studies $[4,19,23]$ involving a total of 211 subjects recorded the prevalence of delirium of participants in the treatment group and the comparison group. The pooled results (pooled RR $=0.49 ; 95 \% \mathrm{CI}, 0.28 \sim 0.88, p=0.017 ; I^{2}=$ $49.3 \%, p$ for heterogeneity $=0.139$ ) showed that exogenous administration of melatonin and melatonin receptor agonists could significantly decrease the prevalence of delirium and no 
Fig. 1 PRISMA flow diagram of literature research and selection process
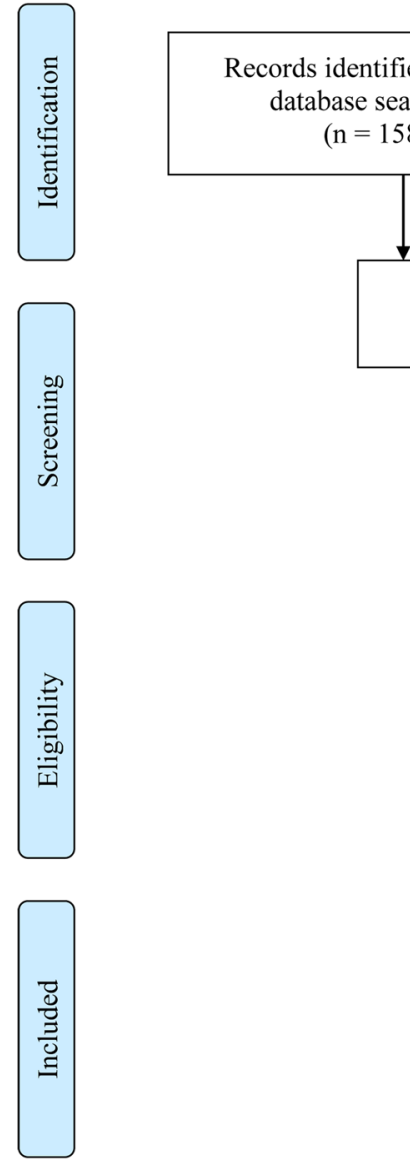

Records after duplicates removed $(\mathrm{n}=184)$

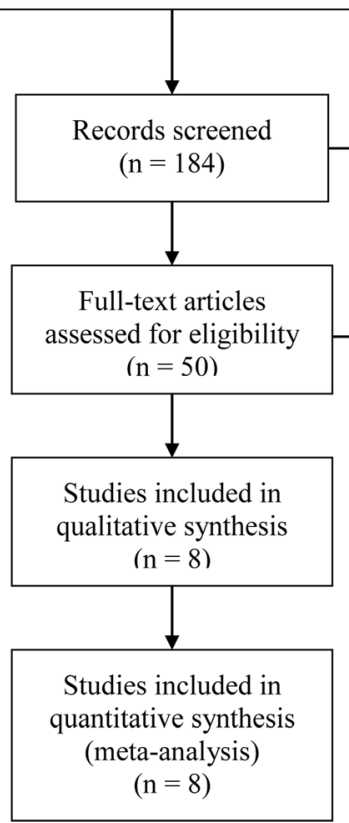

Full-text articles excluded, with reasons:

-Reviews $(\mathrm{n}=17)$

-Irrelevant articles $(n=5)$

-Did not exclusively investigate

melatoninergic agents $(\mathrm{n}=2)$

-Ineligible participants $(n=7)$

- Protocols $(n=6)$

$-\operatorname{Not} \operatorname{RCT}(\mathrm{n}=5)$ significant heterogeneity across studies were identified (Fig. 3a).

\section{Total sleep time}

Six [19, 21-25] studies recorded sleep time of patients in the treatment group and the control group, among which two [19, 23] studies assessed the total sleep time and four [21, 22, 24, 25] paid special attention to the nocturnal sleep time. Definition of time available for nocturnal sleep varied in these articles: $7 \mathrm{~h}$ (from midnight to $7 \mathrm{am}$ ) [21], $9 \mathrm{~h}$ (from $9 \mathrm{pm}$ to 6 am) [22], $10 \mathrm{~h}$ (from $9 \mathrm{pm}$ to $7 \mathrm{am}$ ) [24], and $8 \mathrm{~h}$ (from $10 \mathrm{pm}$ to $6 \mathrm{am}$ ) [25]. Bourne et al. [24] provided four kinds of total nocturnal sleep time by using different assessment methods (bispectral index (BIS) [below 80], actigraphy, nurse assessment, and patient assessment), among which actigraphy and nurse assessment were the common methods adopted by other studies while bispectral index, an updated signal-processing technique based on the electroencephalogram (EEG), was prioritized in this study and considered as a primary analysis. Three studies $[21,23,25]$ rated sleep time by observation of beside nurses or raters, one [19] by retrospectively reviewing the electronic charts, and one [22] by using a standard procedure described by Rechtschaffen and Kales [26]. The combined results (pooled WMD $=0.43 ; 95 \%$ CIs, $0.02 \sim 0.88, p=0.063 ; I^{2}=68.6 \%, p$ for heterogeneity $<0.01$ ) showed that exogenous administration of melatonin and melatonin receptor agonists was associated with a trend towards longer (although not statistically different) duration of sleep (Fig. 4a). Subgroup analysis also revealed an increased but not statistically significant duration of sleep in the treatment group and insignificant heterogeneity across studies after omittance of the study conducted by Bourne and his colleagues [24] (pooled WMD $=0.29 ; 95 \% \mathrm{CI},-0.01 \sim 0.60, p=0.059 ; I^{2}=$ $2.3 \%, p$ for heterogeneity $=0.393$ ).

Meanwhile, if we adopted sleep time obtained from the more traditional secondary analysis (actigraphy and nurse assessment) of Bourne et al.'s study, the pooled WMD was listed as below: $0.18\left(95 \% \mathrm{CI},-0.12 \sim 0.48, p=0.028 ; I^{2}=19.8 \%, p\right.$ for heterogeneity $=0.284)$ and $0.11(95 \% \mathrm{CI},-0.27 \sim 0.49$, $p=0.572 ; I^{2}=46.1 \%, p$ for heterogeneity $\left.=0.099\right)($ Fig. S1a and c). These results were stable in sensitivity analyses when omitting studies one by one (Fig. S1b and d). Patients' subjective assessment may lead to reporting bias due to the inaccuracy in recording their time to fall asleep and wake up. Statistical analysis supported this view, indicating significant heterogeneity among this set of data and data provided by other studies with a pooled WMD of 0.03 (95\% CI, - 


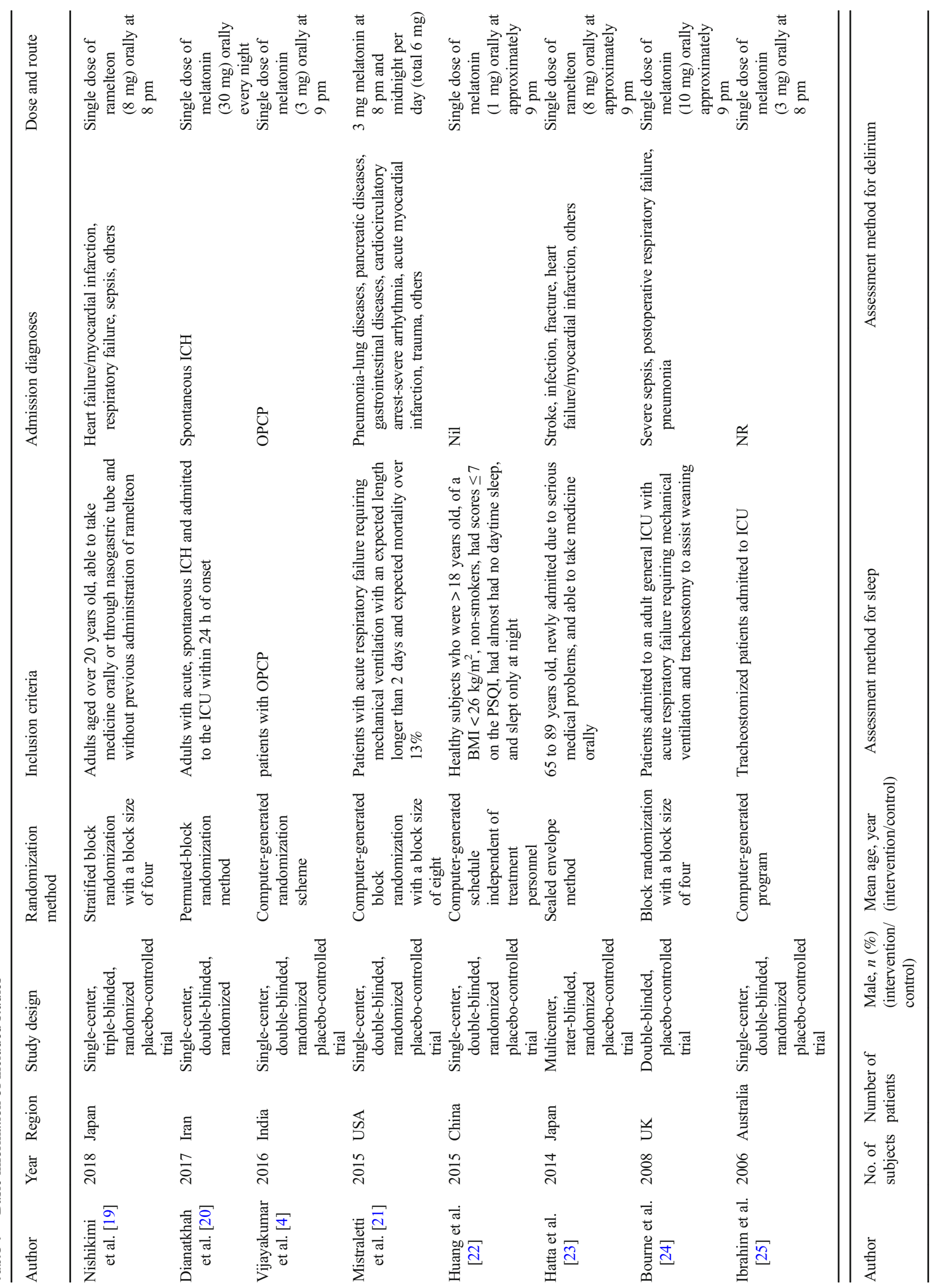




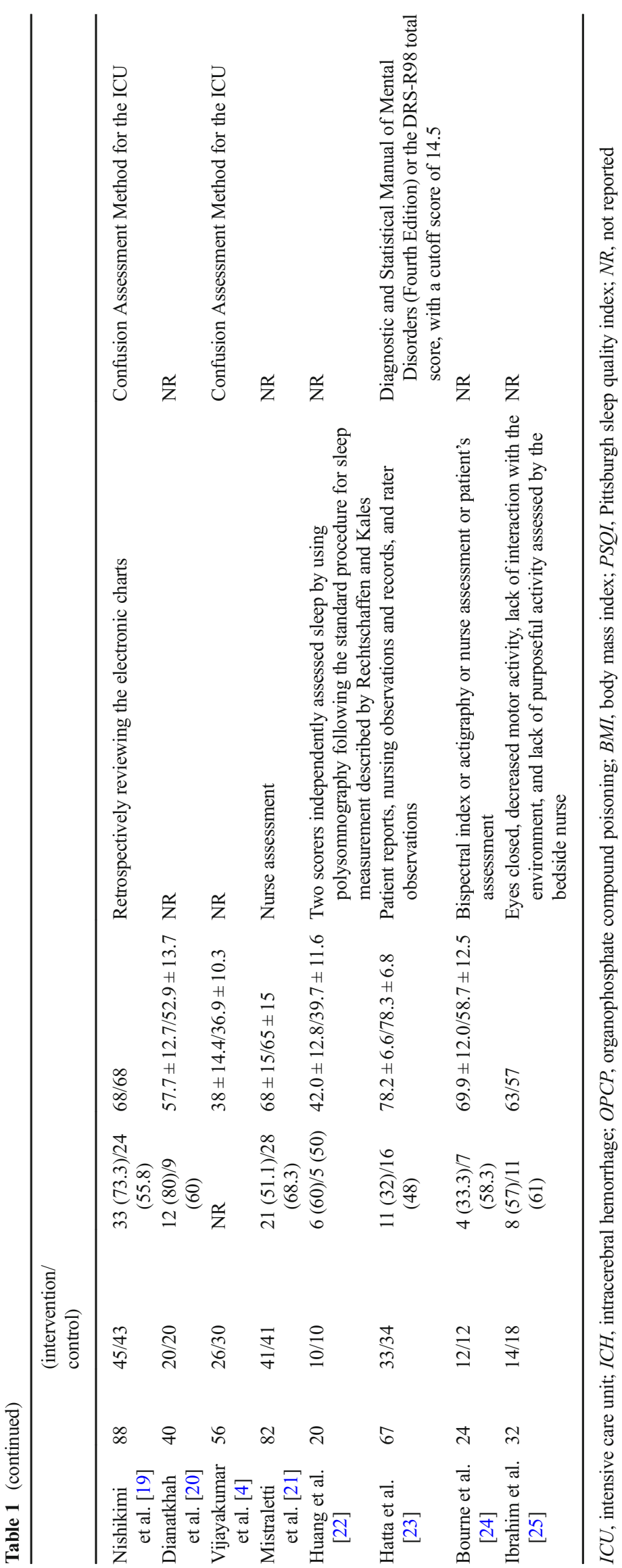


$0.48 \sim 0.53, p=0.92 ; I^{2}=72.6 \%, p$ for heterogeneity $=0.003$ ) (Fig. S1e). Sensitivity analysis adopted sleep duration data from patients' assessment in Bourne et al.'s study was not conducted due to the significant heterogeneity.

\section{Number of awakenings per nights}

Three studies $[19,22,23]$ recorded the number of awakenings per night. The combined results (pooled $\mathrm{WMD}=-2.03 ; 95 \%$ CI, $-3.83 \sim 0.22, p=0.028 ; I^{2}=93.9 \%, p$ for heterogeneity $<0.001$ ) showed that exogenous administration of melatonin and melatonin receptor agonists could significantly decrease the number of awakenings per night (Fig. 4b). Subgroup analysis showed that the majority of heterogeneity came from different types of used agents (melatonin or ramelteon) and administration of ramelteon could also decrease the number of awakenings per night (pooled $\mathrm{WMD}=-0.46 ; 95 \% \mathrm{CI}$, $0.78 \sim-0.14, p=0.005 ; I^{2}=0.0 \%, p$ for heterogeneity < 0.001 ) (Fig. 4b) $[19,23]$.

\section{Duration of mechanical ventilation}

One [4] study provided the accurate mean and SD for the duration of mechanical ventilation; two [20,21] provided the medians and interquartile ranges for the duration of mechanical ventilation, and we estimated the means and SDs by using Wan and Luo's methods $[16,17]$. The pooled results (pooled $\mathrm{WMD}=-1.86 ; 95 \% \mathrm{CI},-4.03 \sim 0.31, p=0.092 ; I^{2}=$ $0.0 \%, p$ for heterogeneity $=0.646$ ) showed that exogenous administration of melatonin and melatonin receptor agonists was associated with a trend towards decreased duration of mechanical ventilation but this difference was not statistically significant (Fig. 4c).

\section{Duration of ICU stay}

One [4] study provided the accurate mean and SD for the duration of ICU stay and one [19] study provided logtransformed data of the duration of ICU stay; two [20, 21] presented medians and interquartile ranges for the duration of ICU stay, and we estimated the mean and SD by using Wan and Luo's methods $[16,17]$. The pooled results (pooled $\mathrm{SMD}=-0.32 ; 95 \% \mathrm{CI},-0.56 \sim 0.07, p=0.002 ; I^{2}=0.0 \%$, $p$ for heterogeneity $=0.976$ ) showed that exogenous administration of melatonin and melatonin receptor agonists could significantly decrease the duration of ICU stay (Fig. 4d).

\section{Mortality during ICU}

Three studies $[4,19,23]$ involving a total of 210 subjects recorded mortality at the discharge of ICU in the treatment group and the control group. The pooled results (pooled $\mathrm{RR}=1.07 ; 95 \% \mathrm{CI}, 0.60 \sim 1.90, p=0.823 ; I^{2}=3.3 \%, p$ for heterogeneity $=0.355$ ) showed that exogenous administration of melatonin and melatonin receptor agonists could not significantly change the mortality of patients during their stay in ICU (Fig. 3b). No significant heterogeneity across studies was identified.

\section{Qualitative analysis}

\section{Sleep quality}

Bourne et al.'s study [24] also reported area under the curve (AUC) for BIS, with a decreased AUC presenting better sleep and finally, a decrease of $7 \%$ of AUC was identified in those participants who had melatonin.

\section{Need for sedation}

Mistraletti et al. [21] recruited 82 critically ill patients with mechanical ventilation admitted to ICU and then assigned them to receive either melatonin $(3 \mathrm{mg}+3 \mathrm{mg})$ or placebo with a ratio of 1:1. A lower amount of enteral sedatives and antipsychotics and improved neurological indicators (e.g., pain, agitation, and anxiety) were observed in the treatment group.

\section{Safety and tolerance}

Two studies $[21,24]$ reported adverse events that may relate to the administration of melatonin. Bourne et al. [24] described that one case of headache in the melatonin group was quickly alleviated after the application of acetaminophen; Mistraletti et al. [21] described that one subject in the treatment group had excessive sleepiness and the administration of melatonin had to be discontinued.

\section{Discussion}

Clinical practice guidelines about preventing and managing pain, agitation, and delirium in adult patients in the ICU recommend optimized environment, decreased stimuli, and low level of noise [27]. Currently, although sedatives (benzodiazepines and nonbenzodiazepine hypnotic agents) are widely used to improve sleep quantity and quality for ICU patients as well as to control delirium-associated anxiety and agitation, previous literature clearly proved the lack of efficacy of these drugs [28]. Moreover, sedatives have certain depressed respiratory action, which may prolong the duration of mechanical ventilation $[28,29]$ and act as a risk factor for the occurrence of delirium in ICU patients [27].

Results of now-available studies on the effect of prophylactic use of melatonin in hospitalized elderly or perioperative patients are controversial. Jaiswal et al. [30] refuted the 


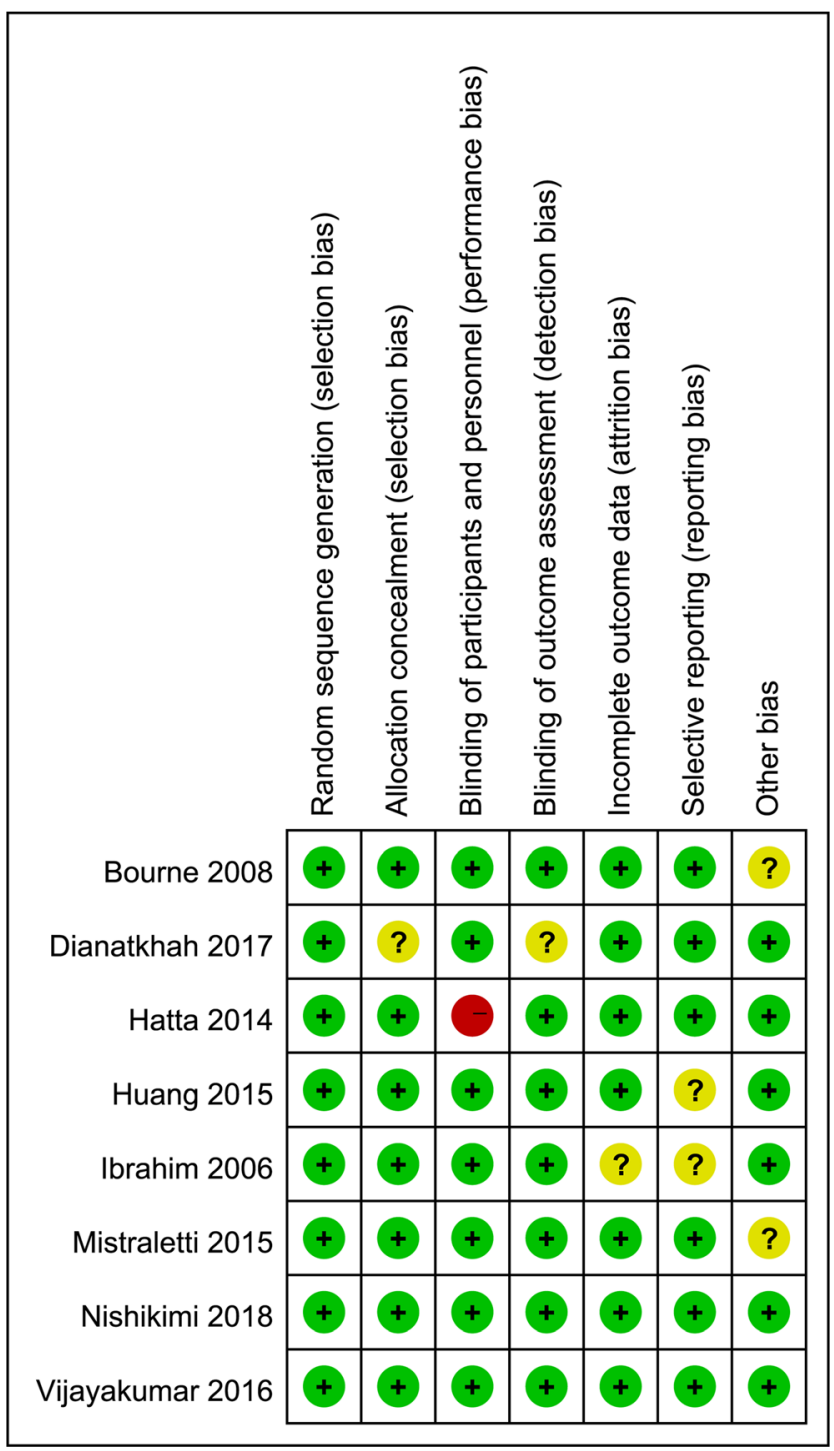

Fig. 2 Summary of authors' judgements about the risk of bias items for each included study; red in the figure indicates high risk, yellow represents unclear risk, and green means low risk

effect of melatonin in improving subjective or objective sleep quality and delirium in hospitalized non-ICU patients older than 65 while Mousavi and his colleagues [31] proposed that melatonin at a dose of $3 \mathrm{mg}$ nightly was effective in decreasing the Pittsburgh Sleep Quality Index (PSQI) score in sulfur mustard-exposed patients. Intensive care unit is a special clinical setting with a high incidence of delirium and sleep deprivation. A previous study conducted by Dessap et al. [5] showed that reduced plasma melatonin levels were associated with unsuccessful and prolonged weaning of ventilator, increased duration of ICU stay, and poor prognosis. To the best of our knowledge, this is the first systematic review and meta-analysis appraising the beneficial effect of exogenous administration of melatonin and melatonin receptor agonists for improving sleep and delirium in subjects in intensive care units.
Among the included studies, the reported incidence of delirium ranged from 3 to $50.85 \%$ in the treatment group and from 32 to $84.81 \%$ in the control group $[4,19,23]$. The pooled results $(R R=0.49)$ suggested that the use of exogenous melatoninergic agents could reduce the incidence of delirium by about half. Melatoninergic agents possess direct antioxidant activity and therefore could neutralize free radicals and downregulate proinflammatory enzymes in the central nervous system [32]. In this study, it could be hypothesized that decreased incidence of delirium in the treatment group was attributed to the improved quality of sleep as well as the neuroprotective effect of melatoninergic agents. Heterogenous prevalence of delirium in the treatment group across the included studies may be due to the inconsistencies in delirium assessment, and as such, the true effect of melatoninergic therapy on this outcome should be interpreted cautiously [33].

Subjects' sleep can be assessed subjectively by using the Richards-Campbell Sleep Questionnaire (RCSQ) and objectively by total or nocturnal sleep time and sleep fragmentation. Although it was not statistically significant, our study showed that the administration of melatonin or ramelteon was associated with a trend towards increased duration of sleep time (about half an hour). This finding needs to be considered as a hypotheses-generating result. Due to frequent awakenings, patients in ICU experience a high proportion of stage 1 and 2 non-rapid eye movement (NREM) sleep with scant restorative slow-wave (SW) and rapid eye movement (REM) sleep [34]. In this study, intervention groups showed decreased numbers of awakenings per night compared with the control groups. Meanwhile, Nishikimi et al. [19] revealed that a higher proportion of nights without awakenings was related to the administration of ramelteon $(51 \%$ vs $30 \%$ for the treatment and the control groups; $p=0.048$ ). These results support that melatonin and melatonin receptor agonists could improve the quality and duration of restful sleep of patients in ICU.

We also examined the effect of melatonin and melatonin receptor agonists on other clinical outcomes of ICU patients. Long-term use of mechanical ventilation may lead to negative outcomes such as lung injury, pneumonia, and sinus [35]. It was extrapolated that the application of melatonin and melatonin receptor agents could diminish the need for sedative agents and therefore shorten the duration of mechanical ventilation. However, in this study, no difference in the duration of mechanical ventilation or mortality was identified between the treatment and the control groups. Due to the heterogenous units and dimension to record duration of ICU stay, we pooled standardized mean difference of individual study for this outcome parameter. Eventually, the duration of ICU stay in the treatment group was reduced by about $30 \%$ in comparison with the control group.

Differing performance of melatoninergic agents in the included studies is possibly due to their various drug doses and population characteristics. Melatoninergic agents exhibit their 
a

Study

ID

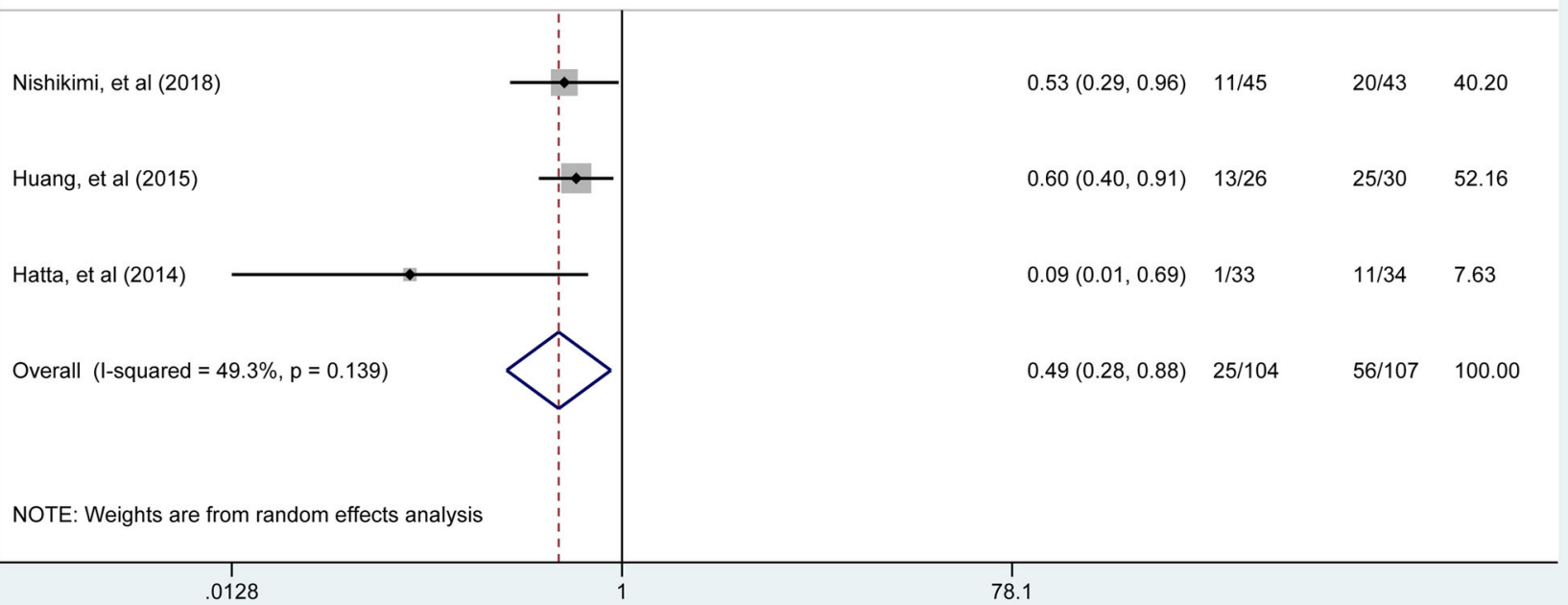

b

Study

ID

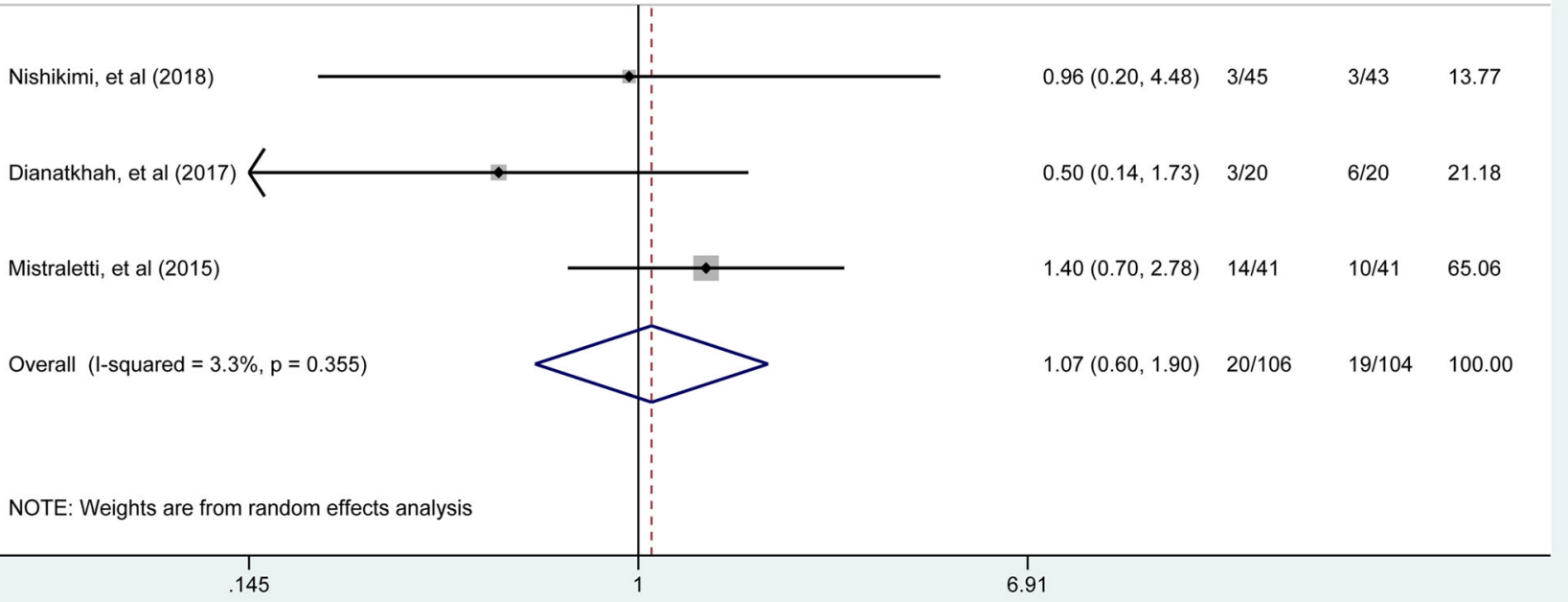

Fig. 3 Forest plot of the comparison about the prevalence of delirium (a) and mortality (b) in-ICU. a The diamond in the left of the central vertical line represents lower prevalence of delirium in the treatment group in

activity by binding membrane melatonin receptors (MT1 and MT2) [36]. Ramelteon is the only selective melatonin receptor agonist approved by the Food and Drug Administration that has been investigated in ICU adult patients, and Miyamoto et al. demonstrated that the affinity of MT1 and MT2 for
$\mathrm{RR}(95 \% \mathrm{Cl}) \quad$ intervention control Weight
Events, Events, \% 


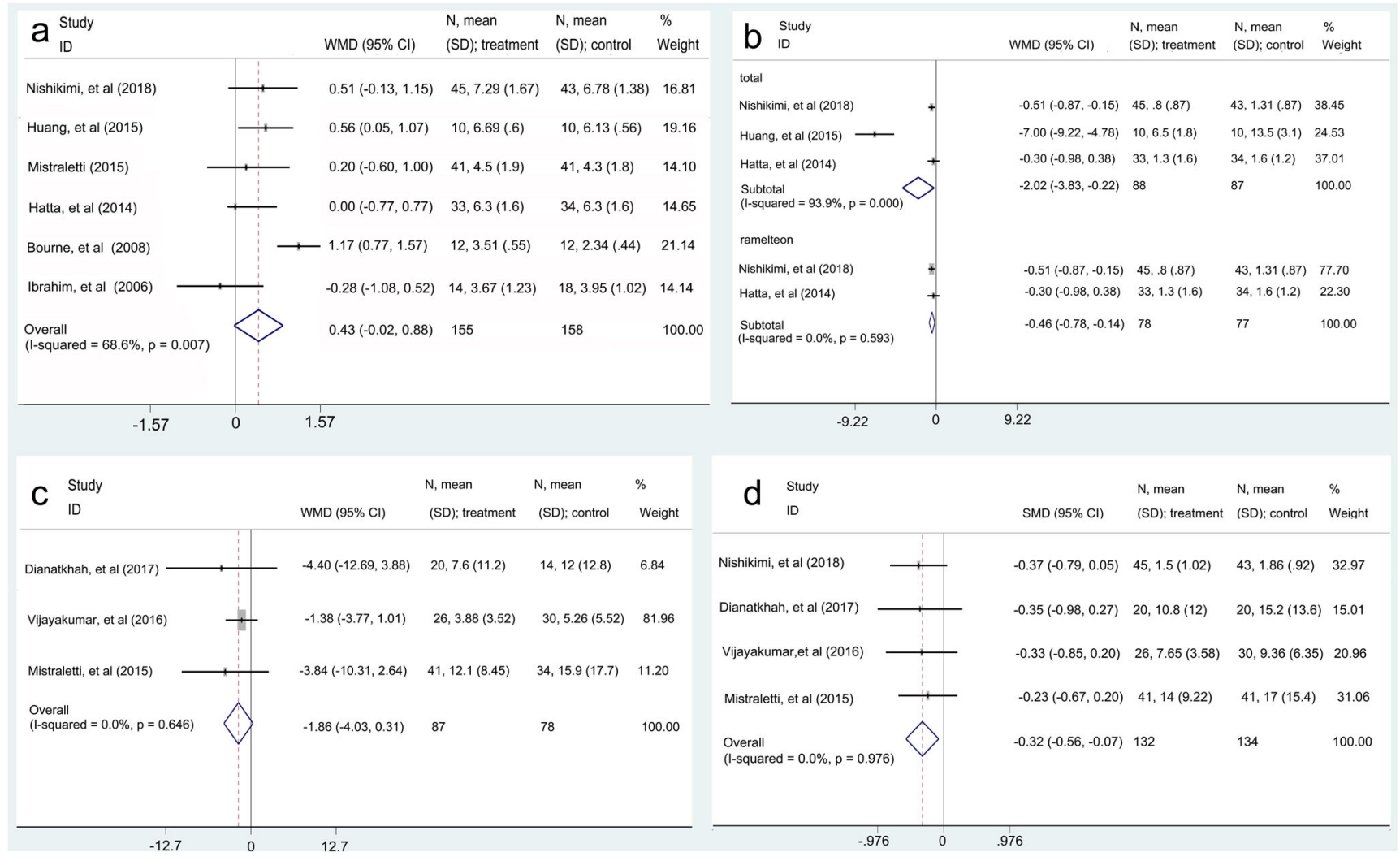

Fig. 4 Forest plot of the comparison about sleep duration (a), the number of awakenings per night (b), duration of mechanical ventilation (c), and duration of ICU stay (d). a The diamond intersecting the central vertical line represents a trend towards longer (but not significantly different) duration of sleep in the treatment group in comparison with the control group. b The diamond in the left of the central vertical line represents decreased number of awakenings per night in the treatment group in

comparison with the control group. c The diamond intersecting the central vertical line represents no differences between the treatment group and the control group. d The diamond in the left the central vertical line represents decreased duration of ICU stay in the treatment group in comparison with the control group; CI, confidence interval; WMD, weighted mean difference; SD, standard deviation

decreased during aging and Alzheimer's disease [38] and it has been widely acknowledged that brain injury would result in lower expression level of MT1 and MT2 [39], which could be a possible explication as to why melatonin is only working in some patients. It was noteworthy that no obvious sideeffects (e.g., abuse, carry-over effect, and cognitive impairment) implicated with the application of melatonin or ramelteon were identified, indicating a potential advantage in terms of safety profile among ICU patients. It would be of great interest to investigate the cost-effectiveness of prophylactic administration of melatoninergic agents in the ICU and therefore better management of delirium and sleep duration with melatonin and ramelteon independent of underlying disease could be translated into clinical benefit.

The strength of our study was increased compared with the previous systematic review presented by Lewis [12] for several reasons. First, eight high-quality randomized controlled trials were included. Although 26 additional records were identified by checking the reference lists of related articles, after reading their abstracts, we did not include them to quantitative analysis, which proved that search strategy of this investigation was reliable. Second, in this study, statistical analysis was conducted to obtain more potent outcome estimates. Third, more endpoints including sleeping time, number of awakenings per night, duration of ICU stay, and mechanical ventilation as well as in-ICU mortality were compared between the treatment group and the control group.

There were also limitations of this study meriting consideration. First, investigators did not distinguish different types of delirium (hyperactive, hypoactive, or mixed-type delirium). Second, due to the small number of studies in the analysis of some parameters, sensitivity analysis might be not available and publication bias (Egger's test or construction of funnel plot) was not assessed. Third, some authors did present means and SDs for continuous data and therefore, estimated means and SDs were employed in the analysis of duration of ICU stay and mechanical ventilation. Last but not least, light is an important point which may influence melatonin secretion but due to limited data, we did not investigate light exposure in this article. 


\section{Conclusion}

In a word, this systematic review and meta-analysis provided robust evidence that prophylactic administration of melatonin or melatonin receptor agonists was associated with a reduced prevalence of delirium, improved quality of sleep, and a trend towards shortened duration of ICU stay in participants in ICU. However, there was no improvement in the duration of mechanical ventilation and in-ICU mortality. Large-scale randomized placebo-controlled studies are still warranted to confirm current results.

\section{Compliance with ethical standards}

Conflict of interest The authors declare that they have no conflict of interest.

Ethical approval For this study, ethical approval is not required.

Informed consent For this type of study, formal consent is not required.

\section{References}

1. Brzezinski A (1997) Melatonin in humans. N Engl J Med 336(3): 186-195. https://doi.org/10.1056/NEJM199701163360306

2. Weinhouse GL (2014) Delirium and sleep disturbances in the intensive care unit: can we do better? Curr Opin Anaesthesiol 27(4): 403-408. https://doi.org/10.1097/ACO.0000000000000093

3. Maldonado JR (2008) Pathoetiological model of delirium: a comprehensive understanding of the neurobiology of delirium and an evidence-based approach to prevention and treatment. Crit Care Clin 24(4):789-856. https://doi.org/10.1016/j.ccc.2008.06.004

4. Vijayakumar HN, Ramya K, Duggappa DR, Veeranna Gowda KM, Sudheesh K, Nethra SS, Raghavendra Rao RS (2016) Effect of melatonin on duration of delirium in organophosphorus compound poisoning patients: a double-blind randomised placebo controlled trial. Indian J Anaesth 60(11):814-820. https://doi.org/10.4103/ 0019-5049.193664

5. Dessap AM, Roche-Campo F, Launay JM, Charles-Nelson A, Katsahian S, Brun-Buisson C, Brochard L (2015) Delirium and circadian rhythm of melatonin during weaning from mechanical ventilation: an ancillary study of a weaning trial. Chest 148(5): 1231-1241. https://doi.org/10.1378/chest.15-0525

6. Mehta S, Cook D, Devlin JW, Skrobik Y, Meade M, Fergusson D, Herridge M, Steinberg M, Granton J, Ferguson N, Tanios M, Dodek P, Fowler R, Burns K, Jacka M, Olafson K, Mallick R, Reynolds S, Keenan S, Burry L, SLEAP Investigators, Canadian Critical Care Trials Group (2015) Prevalence, risk factors, and outcomes of delirium in mechanically ventilated adults. Crit Care Med 43(3):557566. https://doi.org/10.1097/CCM.0000000000000727

7. Harrison Y, Horne JA, Rothwell A (2000) Prefrontal neuropsychological effects of sleep deprivation in young adults-a model for healthy aging? Sleep 23(8):1067-1073

8. Walker CK, Gales MA (2017) Melatonin receptor agonists for delirium prevention. Ann Pharmacother 51(1):72-78. https://doi.org/ $10.1177 / 1060028016665863$

9. Macleod MR, O'Collins T, Horky LL, Howells DW, Donnan GA (2005) Systematic review and meta-analysis of the efficacy of melatonin in experimental stroke. J Pineal Res 38(1):35-41. https://doi.org/10.1111/j.1600-079X.2004.00172.x

10. Maitra S, Baidya DK, Khanna P (2013) Melatonin in perioperative medicine: current perspective. Saudi J Anaesth 7(3):315-321. https://doi.org/10.4103/1658-354X.115316

11. Dianatkhah M, Ghaeli P, Hajhossein TA et al (2015) Evaluating the potential effect of melatonin on the post-cardiac surgery sleep disorder. J Tehran Heart Cent 10(3):122-128

12. Lewis SR, Pritchard MW, Schofield-Robinson OJ, Alderson P, Smith AF (2018) Melatonin for the promotion of sleep in adults in the intensive care unit. Cochrane Database Syst Rev 5:D12455. https://doi.org/10.1002/14651858.CD012455.pub2

13. Mo Y, Scheer CE, Abdallah GT (2016) Emerging role of melatonin and melatonin receptor agonists in sleep and delirium in intensive care unit patients. J Intensive Care Med 31(7):451-455. https://doi. org $/ 10.1177 / 0885066615592348$

14. Foreman B, Westwood AJ, Claassen J, Bazil CW (2015) Sleep in the neurological intensive care unit: feasibility of quantifying sleep after melatonin supplementation with environmental light and noise reduction. J Clin Neurophysiol 32(1):66-74. https://doi.org/10. 1097/WNP.0000000000000110

15. Liberati A, Altman DG, Tetzlaff J, Mulrow C, Gotzsche PC, Ioannidis JPA, Clarke M, Devereaux PJ, Kleijnen J, Moher D (2009) The PRISMA statement for reporting systematic reviews and meta-analyses of studies that evaluate healthcare interventions: explanation and elaboration. BMJ 339:b2700

16. Luo D, Wan X, Liu J, Tong T (2018) Optimally estimating the sample mean from the sample size, median, mid-range, and/or mid-quartile range. Stat Methods Med Res 27(6):1785-1805. https://doi.org/10.1177/0962280216669183

17. Wan X, Wang W, Liu J, Tong T (2014) Estimating the sample mean and standard deviation from the sample size, median, range and/or interquartile range. BMC Med Res Methodol 14(135). https://doi. org/10.1186/1471-2288-14-135

18. Higgins JPT, Green S (editors) (2011) Cochrane handbook for systematic reviews of interventions version 5.1.0 [updated March 2011]. The Cochrane Collaboration. Available from http:// handbook.cochrane.org

19. Nishikimi M, Numaguchi A, Takahashi K, Miyagawa Y, Matsui K, Higashi M, Makishi G, Matsui S, Matsuda N (2018) Effect of administration of ramelteon, a melatonin receptor agonist, on the duration of stay in the ICU: a single-center randomized placebocontrolled trial. Crit Care Med 46(7):1099-1105. https://doi.org/10. 1097/CCM.0000000000003132

20. Dianatkhah M, Najafi A, Sharifzadeh M, Ahmadi A, Sharifnia H, Mojtahedzadeh M, Najmeddin F, Moghaddas A (2017) Melatonin supplementation may improve the outcome of patients with hemorrhagic stroke in the intensive care unit. J Res Pharm Pract 6(3): 173-177. https://doi.org/10.4103/jrpp.JRPP 1749

21. Mistraletti G, Umbrello M, Sabbatini G et al (2015) Melatonin reduces the need for sedation in ICU patients: a randomized controlled trial. Minerva Anestesiol 81(12):1298-1310

22. Huang HW, Zheng BL, Jiang L, Lin ZT, Zhang GB, Shen L, Xi XM (2015) Effect of oral melatonin and wearing earplugs and eye masks on nocturnal sleep in healthy subjects in a simulated intensive care unit environment: which might be a more promising strategy for ICU sleep deprivation? Crit Care 19:124. https://doi.org/10.1186/ s13054-015-0842-8

23. Hatta K, Kishi Y, Wada K, Takeuchi T, Odawara T, Usui C, Nakamura H (2014) Preventive effects of ramelteon on delirium: a randomized placebo-controlled trial. JAMA Psychiat 71(4):397403. https://doi.org/10.1001/jamapsychiatry.2013.3320

24. Bourne RS, Mills GH, Minelli C (2008) Melatonin therapy to improve nocturnal sleep in critically ill patients: encouraging results from a small randomised controlled trial. Crit Care 12(2):R52. https://doi.org/10.1186/cc6871 
25. Ibrahim MG, Bellomo R, Hart GK et al (2006) A double-blind placebo-controlled randomised pilot study of nocturnal melatonin in tracheostomized patients. Crit Care Resusc 8(3):187-191

26. Hori T, Sugita Y, Koga E et al (2001) Proposed supplements and amendments to 'A manual of standardized terminology, techniques and scoring system for sleep stages of human subjects', the Rechtschaffen \&amp; Kales (1968) standard. Psychiatry Clin Neurosci 55(3):305-310. https://doi.org/10.1046/j.1440-1819. 2001.00810.x

27. Devlin JW, Skrobik Y, Gelinas C et al (2018) Clinical practice guidelines for the prevention and management of pain, agitation/ sedation, delirium, immobility, and sleep disruption in adult patients in the ICU. Crit Care Med 46(9):e825-e873. https://doi.org/ 10.1097/CCM.0000000000003299

28. Neufeld KJ, Yue J, Robinson TN, Inouye SK, Needham DM (2016) Antipsychotic medication for prevention and treatment of delirium in hospitalized adults: a systematic review and meta-analysis. J Am Geriatr Soc 64(4):705-714. https://doi.org/10.1111/jgs.14076

29. Jarman A, Duke G, Reade M, Casamento A (2013) The association between sedation practices and duration of mechanical ventilation in intensive care. Anaesth Intensive Care 41(3):311-315

30. Jaiswal SJ, McCarthy TJ, Wineinger NE et al (2018) Melatonin and sleep in preventing hospitalized delirium: a randomized clinical trial. Am J Med 131(9):1110-1117. https://doi.org/10.1016/j. amjmed.2018.04.009

31. Mousavi SS, Shohrati M, Vahedi E, Abdollahpour-Alitappeh M, Panahi Y (2018) Effect of melatonin administration on sleep quality in sulfur mustard exposed patients with sleep disorders. Iran J Pharm Res 17(Suppl):136-144

32. Watson N, Diamandis T, Gonzales-Portillo C, Reyes S, Borlongan CV (2016) Melatonin as an antioxidant for stroke neuroprotection. Cell Transplant 25(5):883-891. https://doi.org/10.3727/ $096368915 \times 689749$

33. Ely EW, Inouye SK, Bernard GR, Gordon S, Francis J, May L, Truman B, Speroff T, Gautam S, Margolin R, Hart RP, Dittus R
(2001) Delirium in mechanically ventilated patients: validity and reliability of the confusion assessment method for the intensive care unit (CAM-ICU). JAMA 286(21):2703-2710

34. Elliott R, McKinley S, Cistulli P, Fien M (2013) Characterisation of sleep in intensive care using 24-hour polysomnography: an observational study. Crit Care 17(2):R46. https://doi.org/10.1186/ $\mathrm{cc} 12565$

35. Ward D, Fulbrook P (2016) Nursing strategies for effective weaning of the critically ill mechanically ventilated patient. Crit Care Nurs Clin North Am 28(4):499-512. https://doi.org/10.1016/j.cnc.2016. 07.008

36. Johnsa JD, Neville MW (2014) Tasimelteon: a melatonin receptor agonist for non-24-hour sleep-wake disorder. Ann Pharmacother 48(12):1636-1641. https://doi.org/10.1177/1060028014550476

37. Miyamoto M (2009) Pharmacology of ramelteon, a selective MT1/ MT2 receptor agonist: a novel therapeutic drug for sleep disorders. CNS Neurosci Ther 15(1):32-51. https://doi.org/10.1111/j.17555949.2008.00066.x

38. Wu YH, Zhou JN, Van Heerikhuize J, Jockers R, Swaab DF (2007) Decreased MT1 melatonin receptor expression in the suprachiasmatic nucleus in aging and Alzheimer's disease. Neurobiol Aging 28(8):1239-1247. https://doi.org/10.1016/j.neurobiolaging.2006. 06.002

39. Osier ND, Pham L, Pugh BJ, Puccio A, Ren D, Conley YP, Alexander S, Dixon CE (2017) Brain injury results in lower levels of melatonin receptors subtypes MT1 and MT2. Neurosci Lett 650: 18-24. https://doi.org/10.1016/j.neulet.2017.03.053

Publisher's note Springer Nature remains neutral with regard to jurisdictional claims in published maps and institutional affiliations. 